\title{
The Implementation of Wideband Cyclostationary Feature Detector with Receiver Constraints
}

\author{
Ikedieze Gabriel Anyim, John Chiverton, Misha Filip and Abdulkarim Tawfik \\ University of Portsmouth, United Kingdom. \\ Email: gabriel.anyim@port.ac.uk
}

\begin{abstract}
Cognitive radio system is a context-aware technology in communications. Spectrum sensing is an important function in the implementation of cognitive radio systems by detecting the presence or absence of primary users within the frequency spectrum and makes available free channels for secondary users. Cyclostationary Feature Detector is capable of detecting signals at low signal to noise ratios relying on the signals features such as cyclic frequency, symbol rate, and carrier frequency and modulation type. Local oscillator frequency offsets, Doppler effects and jitter produce cyclic and sampling clock offsets at the receiver which will degrade the performance. We propose a multi-slot cyclostationary feature detector that reduces the effects of the offsets by deriving the pair of slot and fast Fourier transform sizes that can be used for implementation. Consequently, this pair was used to show that these offsets can be reduced and also compared the detection performance scenarios with and without offsets.
\end{abstract}

Keywords - cognitive radio, spectrum sensing, cyclostationary, cyclic autocorrelation function, spectral correlation function, wideband, feature, detection.

\section{INTRODUCTION}

Radio frequency spectrum is a physical resource that is naturally limited in availability. This calls for its efficient utilization in order to provide users with different services at higher data rates. There is increasing demand for the spectrum due to rapidly expanding market of wireless broadband and multimedia users, and applications which require high data rates. However, these radio frequencies are getting scarce due to spectrum segmentation and dedicated frequency allocation of the standardized wireless systems. As these demands continue to increase, efficient spectrum usage is now a critical issue. On the other hand, several spectrum occupancy measurement campaigns carried out at different parts of the world indicate that a significant amount of the wireless spectrum are under-utilized over a wide range of radio frequencies [1] . Federal Communications Commission (FCC) United States survey measurements indicated that several licensed frequency bands are passive up to ninety percent of the time [2]. Currently, wireless networks are regulated by fixed spectrum assignment policies, where the spectrum is regulated by governmental agencies such as Office of Communications (OFCOM) in United Kingdom (UK). Frequencies are allocated to license holders mostly on long term basis for large geographical regions. The diverse scarcity of communication spectrum has become one of the major issues for the development of new communication systems. In this context, Cognitive Radio (CR) which has context-aware capability and can adapt to its surrounding communication environment has emerged as a promising solution to address the spectrum scarcity by exploring spectral opportunities and deliver a more efficient utilization of the available spectral resources [3]. Cognitive radio concept can be divided into four main blocks or functional components namely: spectrum awareness which aims at determining spectrum availability and the presence or absence of licensed users (primary or incumbent users), spectrum management predicting the length of time the spectrum holes (unused bandwidth or vacancies) will be available for the secondary user before it is released to the primary user [4], spectrum sharing allocates the spectrum holes among the secondary users according to demand and spectrum mobility maintains hitless or error free (seamless) communication during frequency allocation to or from the primary and secondary users thus producing better spectrum transition [5].

This research is on the spectrum sensing block of the CR. Some spectrum sensing techniques such as Energy Detectors and Matched Filter are not sensitive enough to detect the signals at low signal to noise ratio (SNR) and provide correct information to the secondary users [6], [5] and [7]. On the other hand, Cyclostationary Feature Detector (CFD) detects the signals at low SNR using the features of modulated signals such as spectrum and cyclic frequencies and can be applied in a wideband scenario reflecting the real world communications environment. Some literatures have looked at wideband cyclostationary feature detector as in [8] and [6] from different perspectives. In this paper, we propose a multi-slot wideband cyclostationary feature detector using spectral correlation function (SCF) and window-based Fast Fourier Transform (FFT) to remedy the effects of receiver constraints namely: sampling clock offset (SCO) and cyclic frequency offset (CFO). The rest of this paper is divided into 3 sections as follows: In section II, we looked at the underlying principles of spectral correlation function as applicable to CFD and simulated the effects of sampling clock offset (SCO) and cyclic frequency offset (CFO) on the received signal. In section III, the proposed system model was introduced and analyzed including the SCO and CFO. This was followed by more simulations in section IV to arrive at the required pair of slot and FFT needed to reduce the effects of the offsets. 


\section{CYCLOSTATIONARY FEATURE DETECTION}

This section explains the fundamental principles used for feature detection and the receiver impairments affecting the performance of CFD.

\section{A. Spectral Correlation Function}

Autocorrelation function (AF) of a signal $x(t)$ is the similarity between its observations as a function of the time lag between them and is periodic in time $t$ with period $T$. A process shows cyclostationarity if its Autocorrelation function and mean are periodic. According to [9] and [10], the AF can be represented by Fourier series as:

$$
R_{x}\left(t+\frac{\tau}{2}, t-\frac{\tau}{2}\right)=\sum_{\alpha} R_{x}^{\alpha} \tau e^{j 2 \pi \alpha t},
$$

where $\tau$ is the delay and $\alpha$ is the cyclic frequency at which the cyclostationary feature or second order periodicity of the signal occurs. $R_{x}^{\alpha}(\tau)$ are the Fourier series coefficients and give the generalized Cyclic Auto-correlation Function (CAF) shown in $(2)$,

$$
\left.R_{x}^{\alpha}(\tau)=\frac{1}{T} \int_{-T / 2}^{T / 2} R_{x}\left(t+\frac{\tau}{2}, t-\frac{\tau}{2}\right) e^{-j 2 \pi \alpha t}\right) \mathrm{d} t
$$

And can be symmetrically expressed as,

$$
R_{x}^{\alpha}(\tau)=\lim _{T \rightarrow 0} \frac{1}{T} \int_{T} x\left(t+\frac{\tau}{2}\right) x\left(t-\frac{\tau}{2}\right) e^{-j 2 \pi \alpha t} \mathrm{~d} t
$$

where $T$ is the fundamental period and for a modulated signal it is a function of the symbol period and carrier frequency. When $\alpha$ is zero, (3) gives the conventional Cyclic Autocorrelation Function (CAF) whose Fourier transform (FT) is the Spectral Correlation Function (SCF) for the observation time $T^{\prime}$ and expressed as,

$$
S_{x}^{\alpha}(f)=\lim _{1 / T^{\prime} \rightarrow 0} \lim _{T \rightarrow \infty} \frac{1}{T^{\prime}} \int_{-T^{\prime} / 2}^{T^{\prime} / 2} \frac{1}{T} X_{T}\left(t, f+\frac{\alpha}{2}\right)
$$

$$
\times X_{T}^{*}\left(t, f-\frac{\alpha}{2}\right) \mathrm{d} t
$$

where $X_{T}(t, f)$ is the complex envelope of the narrow-band spectral component or short-time Fourier Transform of $x(t)$ with centre frequency $f$, period $T$ (FT length), bandwidth $1 / T$ (of the FT) and cyclic frequency $1 / T^{\prime}$. Further expressed as,

$$
X_{T}(t, f)=\int_{t-T / 2}^{t+T / 2} x(v) e^{-j 2 \pi f v} \mathrm{~d} v .
$$

Given $N^{\prime}$ number of samples, the SCF can be represented discretely as shown in (6),

$$
S_{x}^{\alpha}(n, f)=\frac{1}{N^{\prime}} \sum_{n=0}^{N^{\prime}-1} \frac{1}{L} X_{L}\left(n, f+\frac{\alpha}{2}\right) X_{L}^{*}\left(n, f-\frac{\alpha}{2}\right)
$$

as discussed in [15] and [12], where $N^{\prime}$ is the sensing time, $X_{L}(l, f)$ is the $L$-point fast Fourier transform (FFT) around the $n^{\text {th }}$ sample with $L$ as the discrete FFT length. We refer to (6) as the conventional discrete Spectral Correlation Function. Cyclostationary Feature Detector (CFD) uses either the CAF or SCF in time and frequency domains respectively to detect cyclic features of the received modulated signal such as cyclic and spectrum frequencies as discussed in [11], [12], [13] and [14].

\section{B. Effect of Cyclic Frequency and Sampling Clock Offsets on Spectral Correlation Function}

Cyclic frequency $\alpha$, is a function of both symbol rate $1 / T$ and carrier frequency multiples $2 f_{c}$, where $f_{c}$ is the carrier frequency and $T$ is the symbol period set by the clock at the transmitter [15] and [12]. Every clock produces some errors and in a local oscillator this results to frequency offsets as discussed in [14]. Doppler shift also introduces some uncertainties in the cyclic frequency. Cyclostationary Feature Detector (CFD) requires the knowledge of the cyclic frequency and symbol rate to correctly detect the signals. Therefore, sub-optimal knowledge of cyclic frequency, symbol rate and carrier frequency results to cyclic frequency offset (CFO) at the receiver and it has been shown that the detection performance of CFD is affected as the number of samples increases [14] and [16]. Let the CFO be represented by $\alpha_{\Delta}$. Given that,

$$
\alpha^{\prime}=\alpha\left(1+\alpha_{\Delta}\right)
$$



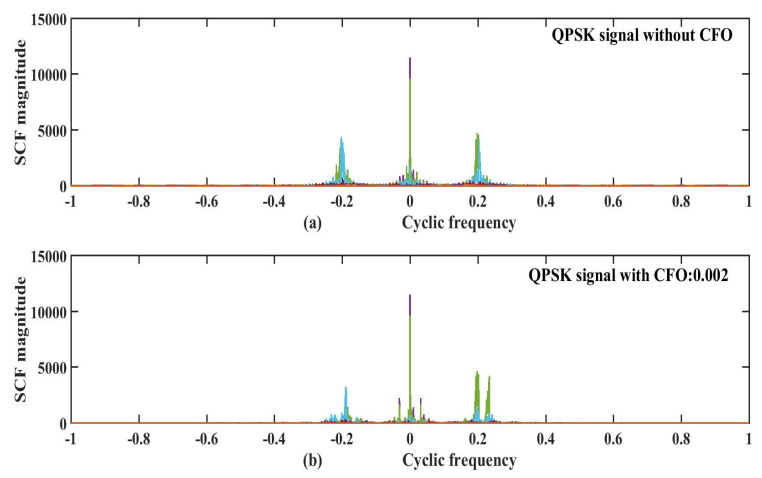

Fig. 1. The Effects of Receiver Offsets on the location of the QPSK signal. (a) SCF of QPSK signal without CFO. (b) SCF of QPSK signal with CFO.

where $\alpha^{\prime}$ is the ideal cyclic frequency (CF) at the Receiver and $\alpha$ is the actual $\mathrm{CF}$ at the transmitter. Some signals have cyclic frequencies at both symbol rate and carrier frequency. The effect of CFO can be determined by substituting (7) for in SCF (4). Some studies have been carried out on the reduction of the impacts of CFO on the CFD using the Cyclic Autocorrelation Function (CAF) for analysis as discussed in [16]. The use of spectral correlation function (SCF) and fast Fourier transform (FFT) to remedy the effects of $\mathrm{CFO}$ and SCO has not been fully explored. Analysis in frequency domain with FFT is less complex and computationally efficient as compared with time domain [17] and hence the approach of this research. Sampling Clock Offset (SCO) is another condition that affects feature detection. It occurs from the frequency offset produced by oscillators and insufficient knowledge of the symbol rates at the Analogue to Digital (A/D) stage of the receiver. Sampling frequencies are produced by these oscillators, with SCO $\delta$, and results in a drift in sampling times and this time-shift varies as the number of samples increases [16], producing phase shift in frequency domain and affecting the cyclic frequency. SCO can be stated as in (8),

$$
T_{s}=(1+\delta) T_{s}^{\prime}
$$

where $T_{s}$ is the sampling period used at the receiver, $T_{s}^{\prime}$ is the actual sampling period with good knowledge of the symbol rate at the transmitter and $\delta$ is the SCO. In order to adequately represent a signal, the sampling rate $1 / T_{\text {symbol }}$ is in multiples of the symbol rate, where $T_{\text {symbol }}$ is the symbol period. The model looks at how to remedy these effects so as to improve the possibility of detection through a statistical model using the SCF and FFT-based slot in a wideband scenario. In Fig. 1, the cyclic frequency is being shifted in position as a result of the presence of CFO and this will affect the possibility of correctly sensing the primary user's (PU) signal. Fig. 1(a) shows the SCF of QPSK signal without any CFO while in Fig. 1(b) the presence of CFO shifting the position of the cyclic frequency.

\section{SYSTEM MODEL}

We propose a wideband multi-slot window-based FFT Test statistic model using the spectral correlation function (SCF) in (4) and (6), to detect the primary users signals $x(t)$. The possibilities of reducing the effects of CFO and SCO were considered. The wideband spectrum is firstly downconverted to baseband producing lowpass signals for effective sampling especially for higher frequencies in the order of gigahertz $(\mathrm{GHz})$ unit. Given a wideband of $N_{B}$ samples divided into $N-$ point slots the relationship can be stated as,

$$
P=\frac{N_{B}}{N}
$$

where $P$ and $N$ are the number of slots and samples per slot respectively. Each slot $p$ is of length $T=N T_{s}$, where $T_{s}$ is the nominal sampling period. Since we are detecting across the entire wideband, the $T_{s}$ is applicable to each slot using a wideband oscillator. This is different from a wideband approach where the $T_{s}$ applies to the entire wideband which makes it difficult for the current Analogue to Digital (A/D) to sample or where $T_{s}$ applies to the signals of interest because in real world scenarios, the signals will not be known and therefore makes implementation works more complex. Our approach is also different from multi-channel wideband where each channel is sampled with a different oscillator which is not cost effective. The use of window in the FFT computation reduces spectral leakage as we sample through the wideband. Applying a window to the FFT leads to the computation of the complex envelope in (5) as,

$$
X(n, f, p)=\sum_{\alpha=-N / 2}^{+N / 2} w(\alpha) x(n-\alpha) e^{-j 2 \pi f(n-\alpha) T_{s}}
$$


where $X(n, f, p)$ is the window-based $w(\alpha)$, narrow-band spectral component of the received signal $x(t)$ for the $p^{t h}$ slot. Considering a multi-slot wideband having the same slot size, the Test Statistic (TS) without the receiver constraints can be expressed as,

$$
\overline{S_{x}^{\alpha}}(n, f)=\frac{1}{P} \sum_{p=1}^{P} S_{x}^{\alpha}(n, f, p)
$$

where $S_{x}^{\alpha}(n, f, p)$ is the discrete SCF for the $p^{t h}$ slot of $N$ samples. This is expressed as,

$$
S_{x}^{\alpha}(n, f, p)=\frac{1}{N} \sum_{n=0}^{N-1} \frac{1}{L} X_{L, p}\left(n, f+\frac{\alpha}{2}\right) X_{L, p}^{*}\left(n, f-\frac{\alpha}{2}\right)
$$

where $X_{L, p}(n, f)$ is the $L$-point window-based FFT of the $p^{t h}$ slot around the $n^{t h}$ sample. Therefore, (11) can be written as,

$$
\begin{aligned}
& \overline{S_{x}^{\alpha}}(n, f)=\frac{1}{P} \sum_{p=1}^{P}\left(\frac{1}{N} \sum_{n=0}^{N-1} \frac{1}{L} X_{L, p}\left(n, f+\frac{\alpha}{2}\right)\right. \\
& \\
& \left.\times X_{L, p}^{*}\left(n, f-\frac{\alpha}{2}\right)\right)
\end{aligned}
$$

while $P$ is the number of slots of $N$ size for the wideband over which the SCF is averaged. Equation (13) shows that the Test Statistic (TS) is the correlation of the FFT of the received wideband signal with itself across the wideband on slot basis as in [18], [19], [20] and [21]. It should be noted that the conventional SCF (6) is a form of the proposed model in (13) where $P=$ 1 and $N=N^{\prime}$. With the presence of CFO and SCO, the cyclic frequency $\alpha$ in (13) is replaced with (7) and the Test Statistic becomes,

$$
\bar{S}_{x}^{\hat{\alpha}}(n, f)=\frac{1}{P} \sum_{p=1}^{P}\left(\frac{1}{N} \sum_{n=0}^{N-1} \frac{1}{L} X_{L, p}\left(n, f+\frac{\hat{\alpha}}{2}\right)\right.
$$

$$
\left.\times X_{L, p}^{*}\left(n, f-\frac{\hat{\alpha}}{2}\right)\right)
$$

where $\hat{\alpha}$ is the cyclic frequency affected by the CFO and SCO offsets. The second moment or power of the Test Statistic (14) can be written as,

$$
\begin{aligned}
\left|\bar{S}_{x}^{\hat{\alpha}}(n, f)\right|^{2}=\mid \frac{1}{P} \sum_{p=1}^{P}\left(\frac{1}{N} \sum_{n=0}^{N-1} \frac{1}{L} X_{L, p}\left(n, f+\frac{\hat{\alpha}}{2}\right)\right. & \\
& \left.\times X_{L, p}^{*}\left(n, f-\frac{\hat{\alpha}}{2}\right)\right)\left.\right|^{2}
\end{aligned}
$$

where $X_{L, p}($.$) is the L$-point window-based FFT of the $p^{\text {th }}$ slot around the $n^{\text {th }}$ sample under the receiver constraints. Equation (15) is needed for determining the probability of detection. Since the cyclic frequency $\alpha$ is a function of symbol rate $1 / T_{\text {symbol }}$ and signal frequency $f$, the resolution of the cyclic frequency is determined by the FFT size and in a multi-slot wideband, additionally by the size of the slot $N$. The cyclic frequency resolution $\Delta_{\alpha}$, of the $L$-point FFT can be expressed as,

$$
\Delta_{\alpha}=\frac{1}{T}=\frac{1}{N T_{s}}=\frac{f_{s}}{N}
$$

where $T, T_{s}, N$ and $f_{s}$ are the sensing period, sampling period, number of samples and sampling rate for the FFT-based slot. The objective is to use the Test Statistics in (14) and the second moment in (15) to get the sizes of FFT and slot that will remedy the effects of the mentioned receiver offsets in the wideband.

\section{A. Threshold and Detection}

A binary decision rule of two hypotheses will be adopted in order to detect the signals.

Hypothesis $1 H_{1}$ for noise only,

$$
H_{1}: s(t)=w(t)
$$

Hypothesis $2 \mathrm{H}_{2}$ for signal present,

$$
H_{2}: s(t)=x(t)+w(t)
$$



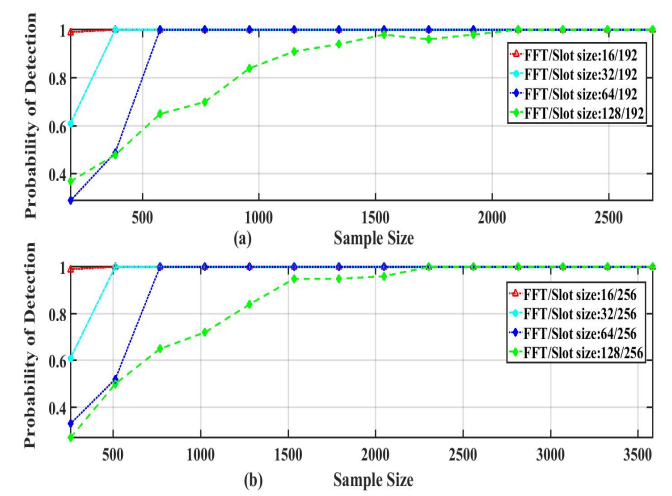

Fig. 2. Probability of Detection with SCO and CFO for multi-FFT sizes.

The threshold that gives constant false alarm rate (CFAR) $T_{\mathrm{d}}$ during the detection is selected. The second moment of the Test Statistics (15) at hypotheses 1 and 2 will be represented with $T S_{1}$ and $T S_{2}$ respectively. Both $T S_{1}$ and $T S_{2}$ will be compared against the detection threshold $T_{\mathrm{d}}$ to determine the presence or absence of the signal as shown below,

- $T S_{1}$ signal is absent, $H_{1}$.

- $T S_{2}$ signal is present, $H_{2}$.

Fundamentally spectral correlation function (SCF) or cyclic autocorrelation function (CAF) is expected to be flat in the presence of noise or exhibits a non-zero mean. From Central limit theorem (CLT) as in [16], [22] and [23], the SCF is Gaussian and Rician distributed. Therefore, the probabilities of false alarm $P_{\mathrm{fa}}$ and detection $P_{\mathrm{d}}$ can be expressed as in (19) and (20) below,

$$
P_{\mathrm{fa}}\left(T_{\mathrm{d}}\right)=\mathbb{Q}\left(\frac{T S_{1 m}}{\sqrt{\sigma_{w}^{2}}}, \frac{T_{\mathrm{d}}}{\sqrt{\sigma_{w}^{2}}}\right)
$$

where $\mathbb{Q}(),. \mathbb{Q}_{w}^{2}$ and $T S_{1 m}$ are the generalized Marcum-Q function, noise variance and the mean of the $T S$ at $H_{1}$,

$$
P_{\mathrm{d}}\left(T_{\mathrm{d}}\right)=\mathbb{Q}\left(\frac{T S_{2 m}}{\sqrt{\sigma_{s}^{2}}}, \frac{T_{\mathrm{d}}}{\sqrt{\sigma_{s}^{2}}}\right),
$$

where $T S_{2 m}$ and $\mathbb{Q}_{s}^{2}$ are the mean and variance of the $T S$ at signal present $H_{2}$ condition.

\section{SIMULATIONS AND RESULTS}

The following simulations were carried out based on (15) and Hanning window for the FFT. In order to remedy the effects of SCO and CFO shown in Fig.1, simulations were carried out to find out the FFT size that delivers the best possible detection using QPSK modulated signal, fixed two slot sizes $N=192$ and 256 samples, fixed sensing time of total samples $N_{B}=3584$ and different FFT sizes of 16, 32, 64 and 128. In Fig. 2, we used QPSK signal at $2.5 \mathrm{MHz}$ frequency $f,-5 \mathrm{~dB}$ signal-to-noise ratio (SNR), sampling rate $f_{s}=1 \times 10^{7}$ and assuming fixed CFO $=\mathrm{SCO}$ at $1 \times 10^{-2}$. It shows that FFT sizes 16 (red) and 32 (cyan) give better probability of detection $P_{\mathrm{d}}$ of the signal with receiver constraints than 64 and 128. It can be observed that sample size up to 500, FFT size 16 (red) produces a higher $P_{\mathrm{d}}$ about 0.95 than 32 and will be used in Fig. 3 to get the matching slot size. The better detection by smaller size FFTs is due to the higher computational efficiency which is in the order of $O(N \log N)$ where $N$ is the FFT size [17]. The lower the $N$, the more computational efficiency the FFT will be. Smaller slot sizes of 128 and 64 were introduced to confirm the best possible slot size that works with the selected FFT 16. In Fig. 3, FFT 16 was used across different slot sizes 64, 128, 192 and 256 to get the better $P_{\mathrm{d}}$. It shows that the smaller the slot size the better the $P_{\mathrm{d}}$. Comparatively, the pair of 16/64 shows higher $P_{\mathrm{d}}$ and will be used to further analyze the model as in Figs. 4-5. Fig. 4 shows the comparison of detection with and without the receiver offsets at different values of SNR with the pair of slot and FFT sizes previously obtained. For each SNR, there is established similarity between the $P_{\mathrm{d}}$ curves in the presence of receiver offsets and that without receiver offsets. This confirms that effects of the receiver constraints have been remedied by deployment of small FFT and slot sizes. It reduces the concern expressed with large samples needed for cyclostationary feature detection under receiver impairments. Fig. 5 is the receiver operating characteristic (ROC) with the slot/FFT size pair $(64,16)$ at different low signal levels with SCO and CFO. Fig. 5 shows the detection performance of the wideband cyclostationary feature detector with receiver constraints. In the presence of receiver constraints, low signal levels up to $-12 \mathrm{~dB}$ SNR are still detectable by the Test Statistic in (14). The proposed model detects much lower level signals under receiver constraints as shown in Figs. 4 and 5 when compared against a time-based model discussed in [16]. It offers lower complexity, speed and accuracy due to the small size FFTs. 


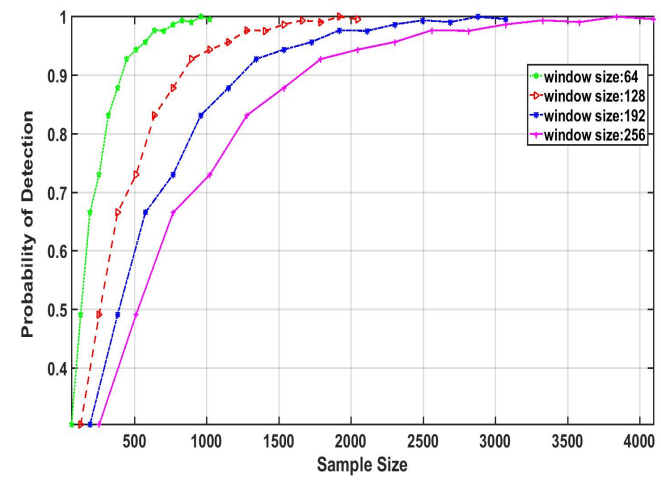

Fig. 3. Probability of Detection in the presence of SCO and CFO with FFT 16 at different slot sizes and -5dB SNR.

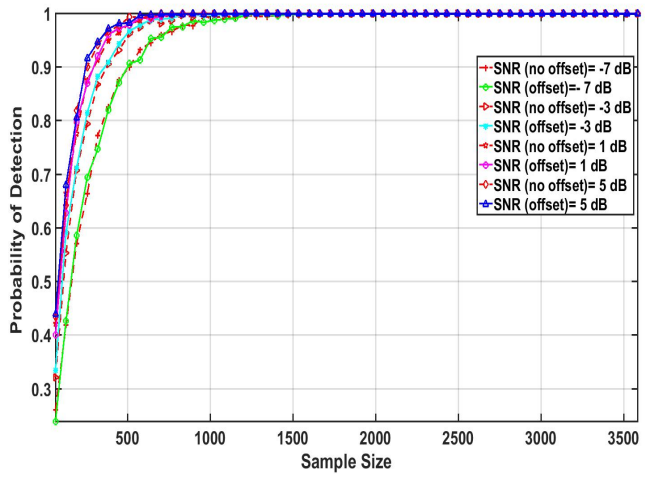

Fig. 4. Probability of detection with the FFT/slot of $16 / 64$ at different SNRs.

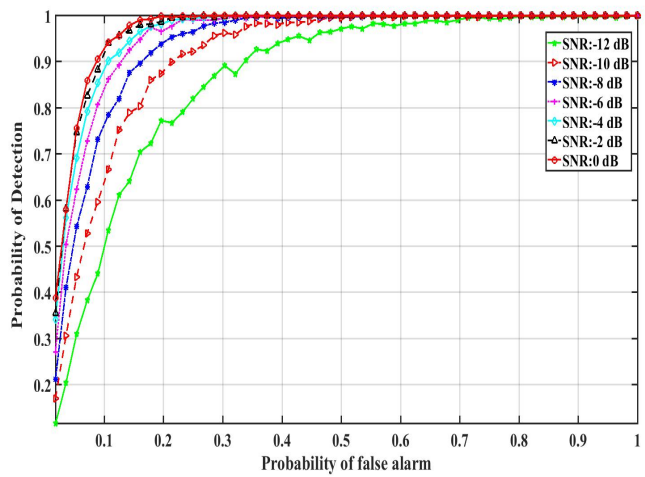

Fig. 5. The Receiver Operating Characteristic at $2.5 \mathrm{MHz}$ QPSK signal at different SNRs with slot-64/FFT-16.

\section{CONCLUSION}

In this paper, we have shown that the effects of sampling clock and cyclic frequency offsets can be reduced by selecting the appropriate small matching pair of slot and fast Fourier transform for the implementation. This is in contrast to the approach of re-sampling the band or the use of large samples considering the cost and computational complexity. This model serves as a guide to the hardware implementation of wideband cyclostationary feature detectors under receiver constraints.

\section{REFERENCES}

[1] K. Patil, R. Prasad, and K. Skouby, "A survey of worldwide spectrum occupancy measurement campaigns for cognitive radio," in International conference on devices and communications (ICDeCom), 2011, pp. 1-5.

[2] P. Kolodzy and et al., "Spectrum policy task force report," Federal Communications Commission,, Washington, USA, ET Docket . 02-135, 2002. 
[3] D. Tarchi and et al, "Technical challenges for cognitive radio application in satellite communications," in IEEE 9th International conference on cognitive radio oriented wireless networks, Oulu, Finland, Jun. 2014, pp. 136-142.

[4] M. Subhedar and G. Birajdar, "Spectrum sensing techniques in cognitive radio networks: A survey," Iinternational journal of next-generation networks (IJNGN), vol. 3, pp. 37-51, Jun. 2011.

[5] T. Yucek and H. Arslan, "A survey of spectrum sensing algorithms for cognitive radio applications," IEEE communications survey \& tutorials, vol. 11, pp. 116-130, Mar. 2009.

[6] D. Cabric, S. M. Mishra, and R. W. Brodersen, "Implementation issues in spectrum sensing for cognitive radios," in IEEE 38th Asilomar Conference on Signals, Systems and Computers, Pacific Grove, USA, Mar. 2004, pp. 772-776.

[7] S. Ren, Z. Zeng, C. Guo, and X. Sun, "Wideband spectrum sensing based on coprime sampling," in IEEE 22nd International conference on telecommunications (ICT), Sydney, Australia, Mar. 2015, pp. 248-352.

[8] D. M. Najafabadi, A. A. Tadaion, and M. R. A. Sahaf, "Wideband spectrum sensing by compressed measurements," in IEEE Computers and Communications Symposium, Cappadocia, Turkey, Jul. 2012, pp. 667-671.

[9] W. A. Gardner, Introduction to random processes with applications to signals and systems. New York: McGraw-Hill, Inc, 1990.

[10] K. Kim and et al, "Cyclostationary approaches to signal detection and classification in cognitive radio," in IEEE New frontiers in dynamic spectrum access networks (DySPAN), Dublin, Apr. 2007, pp. 212-215.

[11] A. Sahai and D. Cabric, "Cyclostationary feature detection," in IEEE Conference on dynamic spectrum access network (DySPAN), California, USA, 2005, pp. 1-69.

[12] W. A. Gardner, "Signal interception: unifying theoretical framework for feature detection," IEEE Transactions on communications, vol. 36, No. 8, pp. 897-906, Aug. 1988.

[13] A. V. Dandawate and G. B. Giannakis, "Statistical tests for presence of cyclostationarity," IEEE Transactions on signal processing, vol. 42, No. 9, pp. 2355-2369, Sep. 1994.

[14] E. Rebeiz, V. Jain, and D. Cabric, "Cyclostationary-based low complexity wideband spectrum sensing using compressive sampling," in IEEE ICC Cognitive radio and networks symposium, Ottawa, ON, Jun. 2012, pp. 1619-1623.

[15] Y. Zeng and Y. Liang, "Robustness of the cyclostationary detection to cyclic frequency mismatch," in IEEE 21st International symposium on personal indoor and mobile radio communications (PIMRC), Istanbul, Sep. 2010, pp. 2704-2709.

[16] E. Rebeiz, P. Urriza, and D. Cabric, "Optimizing wideband cyclostationary spectrum sensing under receiver impairments," IEEE Transactions on signal processing, vol. 61, No. 15, pp. 3931-3943, May 2013.

[17] A. Tkachenko, D. Cabric, and R. W. Brodersen, "Cyclostationary feature detector experiments using reconfigurable bee2," in IEEE 2nd International symposium on new frontiers in DySPAN, Dublin, Ireland, Apr. 2007, pp. 216-219.

[18] K. Po and J. Takada, "Signal detection based on cyclic spectrum estimation for cognitive radio in ieee 802.22 wran system," The institute of electronics, information and communication (IEICE), pp. 1-5, 2007.

[19] D. Yoo, J. Lim, and M. Kang, "Ieee atsc digital television signal detection with spectral correlation density, communications and networks," Communications and networks, vol. 16, No. 6, pp. 600-612, Dec. 2014.

[20] H. Chen, W. Gao, and D. G. Daut, "Spectrum sensing using cyclostationary properties and application to ieee 802.22 wran," in IEEE GLOBECOM, Washington, DC, Dec. 2007, pp. 3133-3138.

[21] Y. Wen-jing, Z. Bao-yu, and M. Qung-min, "Cyclostationary property based spectrum sensing algorithms for primary detection in cognitive radio systems," Springer, pp. 676-680, Dec. 2009.

[22] R. T. Short, "Computation of rice and noncentral chi-squared probabilities," Phaselocked Systems, Technical Report . PHS0254, 2012.

[23] R. S. Roberts, W. A. Brown, and H. H. Loomis, "Computationally efficient algorithms for cyclic spectral analysis," IEEE signal Processing Society, vol. 8, No. 2, pp. 28-49, Apr. 1991. 